\title{
The Bengal Partition of 1905: the Evaluation of British Civilians Activities and Its Effect and Consequence
}

\section{১৯০৫ সালের বঙ্গভঙ্গ: ব্রিটিশ সিভিলিয়ানদের কার্যক্রম এবং বাংলার প্রতিক্রিয়া ও পরিণতি সম্পর্কে একটি পর্যালোচনা}

\section{Debashish Kumar Pramanik $^{1 *}$, Taposh Kumar Neogy ${ }^{2}$}

ISSN: 2311-8636 (Print)

ISSN: 2312-2021 (Online)

Licensed:

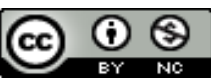

Source of Support: Nil

No Conflict of Interest: Declared

${ }^{*}$ Email for correspondence:

debashishpramanik33@gmail.com
${ }^{1}$ Department of History, University of Rajshahi, Rajshahi-6205, BANGLADESH

${ }^{2}$ Assistant Professor, Institute of Business Administration (IBA), Rajshahi (under National University), BANGLADESH

\section{ABSTRACT}

The Partition of Bengal (1905) and the creation of a new province opened a new chapter in the history of this region. Whatever might have been the real motive of the colonial government behind the scheme, it divided the Hindus and the Muslims of Bengal. Most of the upper caste Hindus opposed it on the ground that by partitioning Bengal the government, in effect, had planned to divide the Bangla- speaking people. The also argued that it was the part of the government's grand design of 'divide and rule'. On the other hand, most of the upper class Muslims in general supported the scheme. The thought that their interests would be better protected in the newly created province and the would be able to overcome decades of backwardness. Yet, there were some Muslims who opposed the partition. As they belonged both to upper class and ordinary section of the Muslim population, their reasons for supporting the partition also varied. Personal, community, national and economic interests prompted interests prompted them to oppose the partition of Bengal.

Keywords: Bengal Partition 1905, British Civilians, Bangladesh

\section{ভূমিকা}

১৯০৫ সালে বঙ্গভঙ্গ বাংলা নামক নতুন প্রদেশে ইতিহাসে এক নতুন অধ্যায় সূচনা করে। ব্রিটিশ উপনিবেশিক সরকারের নীতি ছিল হিন্দু-মুসলিম বিভক্ত রাজনীতি তৈরি করে এই প্রদেশকে শাসন করা। এতে করে উচ্চ শ্রেণীর হিন্দুরা মনে করত ভাষার ভিত্তিতে রচিত এই নতুন প্রদেশ বিভক্ত করে দুর্বল করছে ব্রিটিশ শাসকেরা। যদিও উচ্চ শ্রেণীর হিন্দুদের এই নতুন প্রদেশের বৃহৎ স্বার্থ জড়িত ছিল। তাই তারা তৎকালীণ বঙ্গভঙ্গের বিরোধিতা করেছিল। অন্যদিকে পশচাৎপদ মুসলিম মধ্যবিত্ত শ্রেণী বঙ্গভঙ্গ প্রক্রিয়াকে স্বাগত জানিয়েছিল এই বলে যে, বঙ্গঞ্গের ফলে সৃষ্ট নতুন প্রদেশে অনেক সুযোগ থাকবে। কর্মসংস্থান বাড়বে, কলকারখানা, বিচারালয় স্থাপন’সহ স্বতন্ত্র রাজনীতির সুযোগ পাবে। এই প্রত্যাশায় বঙ্গভঙ্গকে স্বাগত জানায়। কিন্তু প্রগতিশীল 
মুসলিম শ্রেণী বঙ্গভঙ্গের বিরোধিতা করে এই বনে যে, ভাষার ভিত্তিতে রচিত বাংলা নামক নতুন প্রদেশ উপমহাদেশে এক নতুন শক্তি। তাই ১৯০৫ সালের বঙ্গভঙ্গ ও তার প্রতিবাদে স্বদেশী আন্দোলন বাংলার ইতিহালে এক গরুত্বপূর্ন স্থান অধিকার করে আছে। ১৯০৫ সালের ১৬ অক্টোবর বঙ্গভঙ্গ কার্যকর করে ‘পূর্ব বাংলা ও আসাম’ নামক একটি নতুন প্রদেশ সৃষ্টি করা হয়। লর্ড কার্জন ভাইসরয় থাকাকালীন সময়ে (১৮-৯৮-১৯০৫) বঙ্গভঙ্গের ঘটনা ঘটলেও বঙ্গ বিভাগের প্রয়োজনীয়তা অনেক পূর্ব থেকেই অনুভূত হচ্ছিল। বঙ্গভঙ্গের পিছনে প্রশাসনিক না রাজনৈতিক কারণ বেশি দায়ী ছিল সে নিয়ে বিতর্ক আছে। এ প্রসঙ্গে সুমিত কুমার মন্তব্য করেছেন যে, ১৯০৩ পর্যন্ত বঙ্গভঙ্গের পিছনে ব্রিটিশ সরকারের প্রশাসনিক পরিবর্তনের ইচ্ছা কাজ করলেও ডিসেম্বর ১৯০৩ থেকে জুলাই ১৯০৫-এর মধ্যে 'transfer plan was transformed into full scale partition' এ সময়ে রাজনৈতিক অভিলাষ ছিল পূর্ব ও পশ্চিমবঙ্গের বিভক্ত করা। ২ বঙ্গভঙ্গের কারণ বিশ্লেষণ প্রসঙ্গে ম্যাকলেন উল্লেখ করেন যে, ১৯০৫ পর্যন্ত বঙ্গভঙ্গের পিছনে রাজনৈতিক উল্দেশ্য ছিল প্রচ্ছন্ন।’ আমলেশ ত্রিপাঠির মন্তব্য৪:

এ সিদ্ধান্ত আজ বোধ হয় তর্কাতীত যে, রাজদ্রোহী কংগ্রেস কে ধ্বংস করার কোনও উগ্র বাসনা থেকে বঙ্গভঙ্গ পরিকল্পনার জন্ম হয়নি। উনবিংশ শতাব্দীর শেমের দিকে ইংরেজ আমলাদের প্রচন্ড বাঙ্গালি বিদ্বেষ, আর বাংলার ভৌগলিক এবং জনসংখ্যা বৃদ্ধিজাত অতি জরুরী সমস্যা সমাধানের চেষ্টা থেকেই এর উদ্ভব। এসব মন্তব্য থেকে আমরা বলতে পারি যে, প্রথমদিকে প্রশাসনিক প্রয়োজনে বঙ্গভঞ্গের প্রয়োজনীয়তা উপলধ্ধি করা হলেও পরবর্তীকালে এর পিছনে ব্রিটিশ সরকারের রাজনৈতিক উদ্দেশ্যই মুখ্য হয়ে উढে।

\section{ব্রিটিশদের উপমহাদেশে আগমণ}

যোড়শ শতকে ব্রিটিশ বণিকেরা প্রাচ্যের সঙ্গে বাণিজ্য করবার জন্য প্রলুব্ধ হয়ে উতে। প্রায় একশত বছর ধরে পর্তুগীজরা ভারত মহাসাগর এবং প্রাচ্যের ব্যবসা বাণিজ্যের উপর একচেটিয়া কর্তৃত্ব স্থাপন করেছিল। প্রাচ্যের সঙ্গে বাণিজ্য করে পর্তুগীজ বণিকদের প্রচুর মুনাফা অর্জন ব্রিটিশ বণিকরা জানতে পারে। ফলে প্রাচ্যের সঙ্গে লাভজনক ব্যবসায় অংশগ্রহনের জন্য তারা উনুখ হয়ে ওঠে। ১৫৮৮- সালে ব্রিটিশদের হাতে স্পেনের শক্তিশালী নৌবহর ‘স্পেনিজ আর্মাডার’ পতন হলে ব্রিটিশ নাবিক ও বণিকদের মধ্যে প্রচন্ড উৎসাহ দেখা দেয়। ফলশ্রুতিতে ১৫৯৯ সালের ২২ সেত্টেম্বর ব্রিটিশদের কিছু বিশিষ্ট ব্যবসায়ী 'Merchant adventures' নামে একটি বণিক সংঘ গঠন করেন এবং প্রাচ্যের সঙ্গে ব্যবসা বাণিজ্যের জন্য রানী এলিজাবেথের কাছে সনদ প্রার্থনা করেন। ১৬০০ সালের ৩১ ডিসেম্বর রানী এলিজাবেথ বণি সংঘকে প্রাচ্যের সাথে বাণিজ্যের জন্য সনদ মঞ্জুর করেন। সনদ প্রাপ্তির পর এই বণিক সংঘ ‘ইস্ট ইন্ডিয়া কোম্পানী’ নামে পরিচিত হয়।

কোম্পানীকে ১৫ বছরের জন্য ভারতের সাথে বাণিজ্য করার অনুমতি প্রদান করা হয়। রানী স্বয়ং এই কোম্পানীর একজন অংশীদার হন। একজন শাসনকর্তা ও ২৪ জন সদস্য নিয়ে কোম্পানীর কার্যকরী পরিষদ গঠিত হয়। ১৬০৮ সালের সর্বপ্রথম ব্রিটিশরা ভারতে বাণিজ্যকুঠি নির্মাণের উদ্যোগ গ্রহণ করনে পর্তুগীজদের দ্বারা বাধাগ্রন্থ হয় তবুও ক্যান্টেন হকিন্স ব্রিটিশ রাজা প্রথম জেমস্ এর এক পত্র ভারতবর্ষে মুঘল সম্রাট জাহাঙ্গীরের দরবারে আনে এবং সুরাটে বাণিজ্য কুঠি নির্মানে অনুমতি গ্রহণ করে। ১৬১৫ সানে এক নৌযুদ্ধে পর্তুগীজরা ব্রিটিশদের হাতে সম্পূর্ণরূপে পরাজিত হলে মুঘল সম্রাটের কাছ থেকে ব্রিটিশরা ভারতের বিভিন্ন স্থানে বাণিজ্য অনুমতি লাভে সমর্থ হয়। ১৬১৯ সালের মধ্যে সুরাট আগ্রা, আহম্মদাবাদ, ব্রোচ বিভিন্ন স্থানে ব্রিটিশরা বাণিজ্য কুঠি স্থাপন করেন।

\section{ব্রিটিশ সিভিলিয়ানদের প্রভাব বিস্তার ও প্রশাসনিক ক্ষমতা গ্রহণ}

কালের পরিক্রমায় মুঘল শাসকদের দুর্বলতার সুযোগে সমগ্র ভারতবর্টে বাণিজ্যের সাথে সাথে ব্রিটিশরা প্রশাসনিক ক্ষমতা দখলের উদ্যোগী হয়। এর ধারাবাহিকতায় ১৯৫৭ সালে বাংলার শেষ স্বাধীন নবাব সিরাজ-উদ-দৌলা-কে পরাজিত করে ১৭৬৫ সালে ব্রিটিশ বণিক সংঘ দিল্লীর ভূমি রাজস্ব ক্ষমতা গ্রহণ করে। ব্রিটিশ বণিক সংঘের ভারতবর্ষে ভূমি রাজস্বে চরম অব্যবস্থাপনায় ১৭৭০ সালে সমগ্র ভারতবর্ষে দুর্ভিক্ষ দেখা দেয়। এই অবস্থা উত্তরণের জন্য ব্রিটিশ রাজ প্রশাসন ১৭৭৩ সালের ব্রিটিশ বণিক সংঘ নিয়ন্ত্রণের জন্য “The Regulatin Act 1773” পাশ করে। পরবর্তীকালে ভারত উপমহাদেশে ব্রেটিশ রাজপ্রশাসন প্রত্যক্ষভাবে ভারতের ক্ষমতা গ্রহণ করে। এর ধারাবাহিকতায় ব্রিটিশ জনপ্রশাসনের ব্রিটিশ স্বার্থরক্ষাকারী আমলাগণ প্রশাসনিক ক্ষমতা দীর্ঘস্থায়ী করার উল্দেশ্যে ভারতবর্ষকে বিভক্ত করার জন্য বিভিন্ন কর্মপরিকল্পনা ও কার্যক্রম গ্রহণ করে, তার মধ্যে বঙ্গবিভাগ পরিকল্পনা অন্যতম। এই পরিকল্পনা গ্রহনে যেসব বৃটিশ সিভিলিয়ান উদ্যোগী ভূমিকা পালন করেন তাদের মধ্যে উল্লেখযোগ্য ছিলেন ফ্রেজার, রিজলে, অ্যাম্পটহিল, কার্জন ও প্রমুখ।

১৮-৯৮- সালে কার্জন বড়লাট হয়ে আলেন এবং ফ্রেজার ও রিসলের মাধ্যমে ১৯০১-০২ সালে শিক্ষিত বাঙ্গালি সমাজের তথা ভারতে ব্রিটিশ সম্রাজ্যবাদী শাসনকে যারা চ্যালেঞ্জ জানাতে উদ্যত- তাদের বিরুদ্ধে ‘বিভাজন ও শাসনে’র কুপরিকল্পনা রূপায়নের পথে অগ্রসর হন। বঙ্গভঙ্গের প্রস্তাব ‘রিসলে নোটের’ দ্বারা আনুষ্ঠানিকভাবে পেশ করা হয। কার্জনের সহযোগী রিসলে (Risley) 
দু’টি পৃথক নোটে বর্তমানে বহুল পরিচিত সেই চরম সম্রাজ্যবাদী মন্তব্য করে: 'Bengal United is a power. Bengal divided will pull several different ways'। সকলেই বুঝেছিল বঙ্গভঙ্গের সিদ্ধান্ত শুধুমাত্র একটি প্রশাসনিক বা ভৌগোলিক বিভাগ মাত্র নয়; বাঙ্গালি হিন্দু-মুসলমানের মধ্যে মানসিক বিভেদ ঘটানোই এর প্রধানতম উল্দেশ্য- প্রশাসনিক সুবিধার কথা অজুহাত মাত্র।

আপাতদৃষ্টিতে শাসনতান্ত্রিক সুবিধার অজুহাতে বঙ্গ-বিভাগের সিদ্ধান্ত নেওয়া হয়েছিল। কারণ দেখানো হয়েছিল যে বর্তমান বাংলা প্রদেশ অত্যন্ত বড় সুতরাং এই বিরাট প্রদেশ দ্বিখণ্ডিত না হলে সুশাসন সম্ভব নয়। তাছাড়া দুর্গম পূর্ব বাংলাকে কলকাতা থেকে ভালোভাবে শাসন করা সম্ভব নয়। কিন্তু শাসনতান্ত্রিক সুবিধার যুক্তিটি ছিল খুবই অসার। শাসনতান্ত্রিক অজুহাতের আড়ালে বঙ্গভঙ্গের মুল উদ্দেশ্য রাজনৈতিক ও সাম্প্রদায়িক।

কার্জন ছিলেন একজন ঘোরতর সাম্রাজ্যবাদী-তাই বিভিন্ন উপায়ে ভারতীয় জাহীয়তাবাদকে দুর্বল করে তুলতে বদ্ধপরিকর ছিলেন। তিনি লক্ষ্য করেন যে, ভারতীয় জাতীয় আন্দোলনের কেন্দ্রবিন্দু বাংলা এবং বাঙ্গালি হিন্দু-মধ্যবিত্ত নেতারাই সর্বাধিক সক্রিয়। তাই বাংলাকে দ্বিখন্ডিত করে বাঙ্গালি জাতির ঐক্য ও সংহতি নষ্ট করতে পারলে ভারতীয় জাতীয় আন্দোলনের ভিত্তিই দুর্বল হয়ে পড়বে। ব্রিটিশ- ভারত সরকারের এই সাম্প্রদায়িক ভেদনীতি বেশ ফলপ্রসূ হয়ে উঠে ১৯০৪- এর ফেব্রুয়ারিতে কার্জনের পূর্ববঙ্গ সফরের পরে। মুসলিম মধ্যশ্রেণী ও উচ্চবর্গীয় অভিজাত শ্রেণীর সামনে তিনি ও তার সহযোগী ফ্রেজার-ফুলার প্রমুখ উজ্জ্বল ভবিষ্যতের স্বপ্ন তুলে ধরতে সমর্থ হয়। শত বৎসরের মুসলিম পশ্চাদ পদতার কারণে সত্য সত্যই দেশের শিক্ষা, চাকুরী ক্ষেত্রে ও ভূমি ব্যবস্থা এমনকি ব্যবসা-বাণিজ্য (আইন ব্যবসা’সহ) যে হিন্দু ধর্মাবলম্বী শিক্ষিত সমাজের কুক্ষিগত করেছিল সে বিষয়ে সন্দেহের কোন অবকাশ ছিল না। কার্জন এই অসম বিকাশকে সাম্রাজ্যবাদী স্বার্থে কাজে লাগিয়েছিলেন।

\section{ব্রিটিশদের বঙ্গভঙ্গের প্রক্রিয়া ও বঙ্গভঙ্গ}

বঙ্গ বা বাংলা বলতে, ১৯০৫-এর আগে বোঝাত অবিভক্ত বাংলা-বা বেঙ্গল প্রপার, সম্পূর্ণ বিহার, উড়িষ্যা এবং আসাম, এরকম বিস্তৃত একটি অঞ্চল সুষ্ঠুভাবে শাসন করা দুরূহ। প্রধানত এ চিন্তা থেকেই বঙ্গভঙ্গের পঞ্চাশ বছর আগে বাংলার আয়তন কমাবার প্রস্তাব করা হয়েছিল হয়ত এটিও ভাবা হয়েছিলো যে, এতে এ অঞ্চল, বিশেষ করে পূর্ববঙ্গ ও আসানের পশচাৎমখীনতা হ্রাস পাবে।৬ মধ্যপ্রদেশের চীফ কমিশনার আ্যান্ডু ফ্রেজার ও তা-ই মনে করতেন।?

বস্তুতঃপক্ষে বাংলায় পূর্বাঞ্চলের জেলাগুলির এ পশচাৎমুখীতা কুরু হয় মুর্শীদ কুলি খানের সময় থেকে। তিনি ১৭০০ থেকে ১৭২৭ সাল পর্যন্ত প্রথনে বাংলার দেওয়ান ও সুবেদার ছিলেন। মুর্শিদ কুলি খান অষ্টাদশ শতকের গোড়ার দিকে ঢাকা থেকে রাজধানী স্থানান্তর করেন মুর্শিদাবাদে এবং এ পরিপ্রেক্ষিতেই অষ্টাদশ শতকের মাঝামাঝি থেকে পূর্ববঙ্গ (বা ঢাকার) এর ক্ষয় যা থেকে এ অঞ্চলটি বিশ শতকের পূর্ব পর্যন্ত আর মুক্তি পায়নি।

১৮৫৪ সালে, প্রধানত প্রশাসনিক কারনেই বাংলায় লেঃ গভর্ণনরের পদ সৃষ্টি হয়। এরপর ১৮-৬৭ সালে উড়িষ্যার দুর্ভিক্ষের পর বাংলার লেঃ গভর্ণর এক চিঠিতে জানান যে, “বর্তমান বাংলা সরকারের মত এমন অস্বাভাবিক ব্যবস্থা ভারতে আর আছে বনে আমি জানি না, ভারতে আয়তনের দিক থেকে বৃহত্তম প্রশাসনিক ব্যবস্থা আর গুরুত্বের দিক থেকে সর্বপ্রধান হওয়া সত্ত্বেও বাংলা সরকারের কর্মদক্ষতা বোম্বাই ও মাদ্রাস সরকার অপেক্ষা অনেক কম ও দুর্বল।

১৮-৭৪ সালে চীফ কমিশনারের অধীনে আসাম পরিণত হয় একটি স্বতন্ত্র প্রদেশে, ১৮-৬৭ সালে ভারত সচিব লর্ড নর্থকোটের নেতৃত্বাধীন একটি বিশেষ কমিটির সুপারিশে তা কার্যকর করা হয়েছিল। ১৮-৯২ সালে লুসাই উপজাতিদের বিদ্রোহের কারনে আবার বাংলার সীমানা পূর্নগঠনের প্রস্তাব করা হয়েছিলো যদিও তা কার্যকর হয়নি। তবে পূর্ববঙ্গ নিয়ে নতুন প্রদেশ গঠনের প্রস্তাবটি প্রথনে করেন চাটগাঁর কমিশনার ওন্ডহ্যাম ১৮-৯৬ সালে। তিনি সরকারের কাছে এক প্রস্তাবে জানিয়েছিলেন যে, আসাম, চট্টগ্রাম ও ঢাকা বিভাগের অংশ নিয়ে পর্ব বাংলা নামে নতুন প্রদেশ হওয়া উচিত যার রাজধানী হবে চট্টগ্রাম অথবা ঢাকা। অন্যদিকে, এর চার বছর পর আসামের চীফ কমিশনার আসামের সঙ্গে চট্টগ্রাম, ঢাকা ও ময়মনসিংহকে যুক্ত করার প্রস্তাব করেন। তবে ১৮-৯৮- সানে দক্ষিণ লুসাইকে আসামের সঙ্গে যুক্ত করা হয়।ঃ

১৯০৫ সালের বঙ্গভঙ্গ প্রস্তাবটি লড কার্জনের আবিস্কৃত নয় এবং হঠাৎ করেই হয়নি, একথা উল্লেখ করেছেন অনেক বৃটিশ সিভিলিয়ান, হেনরি কটন উল্লেখ করেছেন, ১৯০৫ সালের বঙ্গভঙ্গ অনেক দিনের আলোচনা পর্যালোচনারই ফল ।১০ রোভাট ফ্রেজার মনে করেন, মধ্য প্রদেশের চীফ কমিশনার এ্যান্ড্রু ফ্রেজারের সম্বলপুরের সরকারি ভাষা হিন্দির পরিবর্তে উড়িয়া করার প্রস্তাবের মধ্যেই নিহিত বঙ্গভঙ্গ প্রস্তাব। ১s

ফ্রেজার উল্লেখ করেছেন, বঙ্গভঙ্গের অনেক আগেই পূর্ববঙ্গের দু একটি জেলা আসানের অন্তর্গত ছিল। এবং প্রদেশের বাকি অংশ ‘ট্রান্সফার’ করা ছিল ঢাঁর মতে, 'exceedingly desirable, if not absolutely necessary' কারণ, পূর্ববঙ্গ এক বিরাট অগম্য এলাকা। একজন লেঃ গর্ভণরের পক্ষে এ ভার বহন করা সম্ভব নয়। এর ফলে দেখা যায়, প্রায় ক্ষেত্রে পূর্ববঙ্গের বিভিন্ন 
এলাকা অবহেলিত। অন্যদিকে আসাম এত ক্ষুদ্র যে, তাকে নিয়ে আলাদা একটা প্রদেশ গঠন করা সম্ভব নয়। সুতরাং পূর্ববঙ্গ ও আসাম প্রদেশ হওয়া বাঞ্জনীয়।১২

ফ্রেজারের প্রস্তাবটি পরবর্তী দেড় বছর ধরে বিভিন্ন সিভিলিয়ানরা আলোচনা পর্যালোচনা করেন। পরবর্তীকালে লর্ড কার্জন অনুলোদন করেছিলেন এ প্রস্তাব। সে পরিপ্রেক্ষিতে ১৯০৩ সালের ডিলেম্বরে প্রকাশিত হয়েছিলো রিজলের চিঠি সেখানে আসানের সঙ্গে ঢাকা, চট্টগ্রাম, ময়মনসিংহকে জুড়ে প্রস্তাব করা হয়েছিলো আলাদা একটি প্রদেশ গঠনে।

রিজলের চিঠিটি প্রকাশিত হওয়ার পর থেকেই প্রতিবাদ শুরু হতে থাকে এবং কালক্রমে যা প্রবল আকার ধারণ করে। গর্ভণর জেনারেল নির্বাহী পরিষদের সদস্য ইবেৎসন এক নোটে লিখেছিলেন যে, প্রভাবশালী ব্যক্তিবর্গের ব্যক্তিগত স্বার্থ অপেক্ষা প্রশাসনিক স্বার্থ অনেক বেশী গুরুত্বপূর্ন। এজন্য লর্ড কার্জনকে নিজ পরিকল্পনায় অটল থাকতে বলেন। ১৩ “কার্জন ও ১৯০৪ সালের ১৭ ফেব্রুয়ারি এক নোটে লেখেন, যে কোন ব্যক্তির পক্ষে বাংরার প্রশাসন পরিচালনা এক অসম্ভব ব্যাপার। প্রশাসন পরিচালনার যে দুরূহ ব্যাপার তা অনুধাবনের জন্য তাকে জেলায় যেতে হবে।"১৪

১৯০৪ সালের ১৭ ফেব্রুয়ারি থেকে ২৬ এপ্রিল পর্যন্ত কর্মকর্তাদের মধ্যে এ প্রস্তাব নিয়ে আরও কিছু লেখালেখি হয়। ঢাকার কমিশনার স্যাভ্জে লিখেছিলেন, পূর্ব বাংলাকে এমন প্রদেশ পরিণত করা উচিত যেখানে সম্ভব হবে একটি ব্যবস্থাপক পরিষদ গঠন করা। ফ্রেজার এ প্রদেশে ঢাকা, চট্টগ্রাম কে অন্তর্ভূক্ত করার প্রস্তাব দেন। লর্ড কার্জন এর সঙ্গে পাবনা, বগুড়া ও রংপুরকেও যুক্ত করার প্রস্তাব রাখেন। ১৯০৪ সালের ২২ এপ্রিল বাংলা সরকার অবশেষে সমর্থ হয় একটি পূর্ণাঙ প্রস্তাব তৈরি করতে। ১৫

তবে বঙ্গভঙ্গ প্রস্তাব কিন্তু কার্যকর হয়েছিল ভারাপ্রাপ্ত গভর্ণর জেনারেল এ্যাম্পটহিলের (Ampthill) আমলে। ১৯০৪ সালে ৩০ এপ্রিল কার্জন ইংল্যান্ডে চলে যান ছুটি কাটাতে সাময়িকভাবে কার্যভার গ্রহণ করেন এ্যাম্পটহিল। এ সময় রিজলে নতুন প্রশাসনিক বিভাগে রাজশাহী বিভাগ ও মালদা জেলাকে যুক্ত করার প্রস্তাব করেন। অবশেযে এ সম্পর্কিত প্রতিবেদন গ্রহণ করতে ভারত সচিবের কাছে পাঠিয়ে দেন। কার্জন সচিবকে এ প্রস্তাবের প্রশাসনিক সুবিধাগুলি তুলে ধরেন। ইন্ডিয়া অফিলের সাবেক সচিব গডলেকেও কার্জন উল্লেখ করেন যে, বঙ্গভঙ্গ হচ্ছে “প্রথম শ্রেণীর এক প্রশাসনিক সংস্কার”।৬।

'১৯০৫ সালের ৯ জুন ব্রিটিশ সরকার এ প্রস্তাব অনুমোদন করে এবং ঢাকা, চট্টগ্রাম, রাজশাহী পার্বত্য ত্রিপুরা ও আসাম নিয়ে গঠিত হয় পূর্ববঙ্গ ও আসাম প্রদেশ। ঢাকা হয় নতুন প্রদেশের রাজধানী। এর আয়তন ১,০৬,৫৪০ বর্গমাইন এবং জনসংখ্যা ৩ কোটি ১০ লক্ষ। এর মধ্যে এক কোটি আশি লক্ষ মুসলমান (৫৮\%) এক কোটি বিশ লক্ষ হিন্দু (৩৯\%) এবং অন্যান্য (৩\%)। নতুন প্রদেশে একটি আইন পরিষদ গঠনের বিধান করা হয়, তবে এখানে কলকাতা হাইকোর্টের একতিয়ার বজায় থাকে। বঙ্গভঙ্গ পরিকল্পনা কার্যকর করা হয় ১৯০৫ সালের ১৬ অক্টোবর।’’৭

বঙ্গবিভাগ করার পেছনে প্রশাসনিক যে যুক্তিগুলো ইংরেজ সিভিলিয়ানরা উল্লেখ করেছেন তা একেবারে যুক্তিহীন নয়। সে সময় ও বাস্তবতায় কথা মনে রাখলে ঐসব যুক্তি একেবারে নাকচ করা যায় না। তবে, শেষ পর্যায়ে প্রশাসনিক সুবিধাগুলোর কথা যখন তাঁরা ভাবছিলেন তখন এর সঙ্গে রাজনৈতিক সুবিধাগুলো ও স্পষ্ট হয়ে উঠতে থাকে। এবং যুক্ত হয় রাজনৈতিক আগ্রাসন। কার্জন যখন প্রস্তাবের পক্ষে যে কেউ ছিলেন না তা তো নয়। সেই নির্বাক পক্ষভূক্ত মানুষদের কার্জন চিহ্নিত করতে পেরেছিলেন এবং এ থেকে তিনি রাজনৈতিক সুবিধা নিতে চেয়েছিলেন। ফলে, ব্রিটিশদের সনাতন নীতি ‘Divide and rule policy’ অনুসরণ করা হয়। বঙ্গভঙ্গের রাজনৈতিক দিকটি তখন গুরুত্বপূর্ন হয়ে ওঠে যদিও আমরা লক্ষ করি এ দিকটি গোপন করার ব্যাপারে সিভিলিয়ানরা সতর্ক ছিলেন।

বঙ্গভঙ্গের রাজনৈতিক সুবিধার কথাটি স্পষ্টভাবে উল্লেখ করেছিলেন রিজলে, প্রথম ১৯০৪ সালে। তার মতে, পূর্ববঙ্গে প্রতিবাদ আন্দোলনের নেতৃত্বে আছে শিক্ষিত ক্ষুদ্র একটি গোষ্ঠী এবং ‘বাবু’রা বঙ্গভঙ্গের কারণে বিরোধীতা করা হচ্ছে। তিনি লিখেছিলেন, ঐক্যবদ্ধ বাংলা একটি শক্তি বিভক্ত বাংলা, যা নয়. আমাদের প্রধান উদ্দেশ্য একে বিভক্ত করা এবং এভাবে আমাদের শাসনের বিরুদ্ধে ঐক্যবদ্ধ বিরোধীদের দুর্বল করে তোলা।১b ফ্রেজার ও কার্জন পরে এ বিষয়ে একমত হয়েছিলেন যা বঙ্গভঙ্গ কে ত্বরান্বিত করেছিনো। বঙ্গভঙ্গ প্রস্তাব সংক্রান্ত চিঠিটি প্রকাশিত হওয়ার সঙ্গে সঙ্গে সুরেন্দ্রনাথ বন্দোপাধ্যায় তাঁর পত্রিকায় লিখেছিলেন "We object to the proposed dismemberment of Bengal and we are sure the whole country will rise as one man to protest against it"s৯

\section{বঙ্গভঙ্গের প্রতিক্রিয়া}

বঙ্গভঙ্গের সিদ্ধান্তের পর বাংলার হিন্দু ও মুসলমান সমাজে বিপরীত প্রতিক্রিয়ার সৃষ্টি হয়। কলকাতার প্রভাবশালী শিক্ষিত মধ্যবিত্ত হিন্দু সম্প্রদায় তাৎক্ষনিকভাবে ক্ষুদ্ধ প্রতিক্রিয়া ব্যক্ত করে। কলাকাতার হিন্দু আইনজীবিরা আশঙ্কা করেন যে নতুন প্রদেশের রাজধানী ঢাকায় হাইকোর্ট প্রতিষ্ঠিত হনে তারা পূর্ব বাংলার মক্কেল হারাবেন। সাংবাদিকেরা ভীত হন এই ভেবে যে, নতুন প্রদেশে পত্রপত্রিকা প্রকাশিত হল কলকাতার পত্র পত্রিকার গ্রাহক সংখ্যা কমে যাবে। ব্যবসায়ী সম্প্রদায়ের ভয় ছিল যে, চট্টগ্রাম বন্দর চালু হলে কলকাতা বন্দরে গুরুত্ব হ্রাস পাবে। কলকাতায় বসবাসরত পূর্ববাংলার জমিদার শ্রেণী এই ভেবে আতঙ্কগ্রস্থ হন যে, জমিদারী 
পরিচালনার জন্য তাদেরকে বাসস্থান পরিবর্তন করে ঢাকায় যেতে হতে পারে, কিংবা ঢাকায় আলাদা জমিদারী এস্টাবলিশনেন্ট স্থাপন করার প্রয়োজনীয়তা দেখা দেবে।

কলকাতার শিক্ষিত বাঙ্গালি হিন্দু বুদ্ধিজীবীরা মত প্রকাশ করেন যে, বাংলা বিভাগের মাধ্যমে কলকাতার মর্যাদা খর্ব করা হবে। এভাবে হিন্দু সম্প্রদায়ের সকল পেশাজীবি শ্রেণী বঙ্গবিভাগ কার্যকর হওয়ার পূর্ব থেকেই এর বিরোধিতা শুরু করে। বঙ্গবিভাগের পরিকল্পনা প্রকাশিত হলে সুরেন্দ্রনাথ তাঁর ‘বেঙ্গলি’ পত্রিকায় ‘একটি গভীর জাতীয় বিপদ’ শিরোনানে একটি প্রবন্ধ প্রকাশ করে। বঙ্গভঙ্গের সমালোচনা করে।২০ ‘বেঙ্গলি', ‘হিতবাদী’, ‘সঞ্জীবনী’, প্রভৃতি পত্রিকা বঙ্গভঙ্গ পরিকল্পনার বিরুদ্ধে প্রচার চালাতে থাকে। ২১ সুরেন্দ্রনাথ কলকাতার বাইরের জেলাগুনোতে বঙ্গভঙ্গ বিরোধী প্রচারণা শুরু করেন। ১৯০৩-০৫ সময়কালে বঙ্গভঙ্গ ঠেকানোর কৌশল হিসেবে সভা-সমিতির২২ দ্বারা বঙ্গভঙ্গের বিরুদ্ধে প্রতিবাদ জ্ঞাপন, সংবাদপত্রের মাধ্যমে বঙ্গভঙ্গের সমানোচনা করা, সরকারের নিকট বঙ্গভঙ্গ না করার আবেদন জানানো ইত্যাদি পদক্ষেপ গ্রহণ করা হয়।

১৯০৪ সালের মার্চ মালে এবং ১৯০৫ সালের জানুয়ারি মালে কলকাতার টাউন হলে দুটি প্রতিবাদ সভা অনুষ্ঠিত হয়। মহারাজা মনীন্দ্রচন্দ্র নন্দী, কবি রবীন্দ্রনাথ ঠাকুর প্রমুখ এসব থ্রতিবাদ সভায় যোগ দেন। টাউন হলে অনুষ্ঠিত সভায় বঙ্গভঙ্গের বিরুদ্ধে নিন্দা প্রস্তাব গ্রহণ করে বলা হয় যে, ‘বাঙ্গালিদের ঐতিহাসিক, সামাজিক এবং ভাষাগত ঐক্য ও ঐতিহ্য ছিন্ন করা যাবে না কারণ তাতে বাঙ্গালিদের পার্থিব উন্নতির পথে অন্তরায় সৃষ্টি হবে’।

বঙ্গ বিভাগ না করার আবেদন জানিয়ে যে সকল সংগঠন সরকারের নিকট স্মারকলিপি প্রদান করেছিল সেগুনোর মধ্যে অন্যতম ছিল বেঙ্গল ন্যাশনাল চেম্বার অব কমার্স, ব্রিটিশ ইন্ডিয়ান এসোসিয়েশন, ইন্ডিয়ান মাইনং এসোসিয়েশন প্রভৃতি। এসব সংগঠনের প্রদত্ত স্মারকলিপিতে যুক্তি দেখানো হয় যে, ‘আসাম অনুন্নত এলাকা, তার সঙ্গে বাংলার একাংশের যুক্তকরণ আত্নহত্যারই শামিল, বাংলার শিল্প-বাণিজ্য ক্ষতিগ্রস্থ হবে ইত্যাদি।২৩ জমিদারদের একজন সীতানাথ রায় ও আরো কয়েকজন লে. গভর্নর-এর সঙ্গে দেখা করে বলেছিলে: ‘বঙ্গভঙ্গ হলে তাঁদের সম্পত্তি দুই প্রদেশের অধীনে পড়বে যা জটিলতার সৃষ্টি করবে। তাছাড়া তাদের অধিকাংশ জমিদারী হচ্ছে ঢাকা, ময়মনসিংহ, ফরিদপুর, বাখেরগঞ্জ এবং ত্রিপুরায় এবং সম্প্রতি তারা সম্পত্তি কিনেছেন রাঁচি ও কলকাতায়’।28 কংগ্রেলের নেত্রবৃন্দও বঙ্গভঙ্গের বিরুদ্ধে অবস্থান গ্রহণ করেন। কংগ্রেস নেতৃবৃন্দ বঙ্গবিভাগকে ব্রিটিশদের ‘বিভক্তিকরণের মাধ্যমে শাসন’-এর প্রচেষ্টা এবং ব্রিটিশ বিরোধী বুদ্ধিজীবীদের প্রতি বিদ্বেষের প্রমাণ হিসেবে গণ্য করেন। সকল হিন্দু নেত্রবৃন্দ বঙ্গবিভাগকে 'মাতৃসম প্রদেশে’র অঙ্গ ব্যবচ্ছেদ বনে মন্তব্য করেন। কংগ্রেস বঙ্গভঙ্গের কেবল বিরোধিতাই করেন নি, বঙ্গভঙ্গ রদ করতে আন্দোলনে ঝাপিয়ে পড়ে।

এ আন্দোলনের প্রধান কেন্দ্র ছিল শুরুতে কলকাতা, পরবর্তীতে সারা বাংলায় তা ছড়িয়ে পড়ে। সুরেন্দ্রনাথ বন্দ্যোপাধ্যায়, অরবিন্দ ঘোষ, বিপিন পাল প্রমুখ নেতৃবৃন্দ বঙ্গভঙ্গ বিরোধী আন্দোলনে নেতৃত্ব দেন। ক্রমান্বয়ে এ আন্দোলন বাংলার হিন্দু সম্्্রদায়ের আন্দোলনে পরিণত হয়। ২৫ যেদিন বঙ্গভঙ্গ কার্যকর করা হয় সেদিন (অর্থাৎ ১৬ অক্টোবর) বঙ্গভঙ্গ বিরোধীরা সারা দেশে উদযাপন করে শোক দিবস, পালিত হয় সাধারণ ধর্মঘট, দলে দলে লোক উপবাস করে। নগ্নপদে মিছিল করতে করতে স্বদেশী গানে আকাশ-বাতালে মুখরিত করে তোলে, পরস্পর পরস্পরের হাতে রাখী পরিয়ে দেয় ভ্রাতৃত্বের বন্ধনের প্রতীক হিলেবে। এ আন্দোলনকে সফল করার জন্য কতগুলো কর্মসূচী গ্রহণ করা হয়, যেমন-

ক) দেশী পণ্যের ব্যবহার ও বিদেশী দ্রব্য বর্জন,

খ) স্বরাজ অর্জনের লক্ষ্যে সশস্ত্র সংগ্রানে অবতীর্ণ হওয়া।

অন্যদিকে বাংলার মুসলমান সমাজ ১৯০৪ সালে বঙ্গভঙ্গের প্রস্তাব প্রকাশিত হলে এবং ঢাকা, চট্টগ্রাম ও ময়মনসিংহ জেলাসমূহকে আসাম চিফ কমিশনারের শাসনের অধীনে আনার কথা বলা হলে তাৎক্ষণিক প্রতিক্রিয়ায় ‘মোসলেম ক্রনিকল’ পত্রিকা, সেন্ট্রাল ন্যাশনাল মোহামেডান এসোসিয়েশন এবং কলকাতায় বসবারত নেতৃস্থানীয় মুসলমানদের মধ্যে আব্দুল হালিম গজনভী, আব্দুর রসুল, সৈয়দ ইসমাইল হোলেন সিরাজী প্রমুখ মুসলমান নেতৃবৃন্দ প্রস্তাবিত ব্যবস্থ্যার নিন্দা করেন।২৬ সেন্ট্রাল ন্যাশনাল মোহানেডান এসাসিয়েশনের সম্পাদক সৈয়দ আমীর আলী চিফ সেক্রেটারিকে এক স্মারকপত্রে জানান যে, 'বাংলাভাষী জাতিকে বিচ্ছিন্ন করা যাবে না এবং এর প্রয়োজন ও নেই (.... a readjustment of the territorial limits of Bengal.....was neither necessary nor desirable')। $\left.\right|^{29}$ প্রাথমিকভাবে প্রতিক্রিয়ায় তাদের যুক্তি ছিল প্রস্তাবিত ব্যবস্থায় অনুন্নত আসানের সঙ্গে পূর্ব বাংলার এসব মুসলমান সংখ্যাগরিষ্ঠ জেলা অন্তর্ভূক্ত করা হলে মুসলমানদের কোন লাভ হবে না। কিন্তু মুসলমানদের এই বিভক্ত বিরোধী প্রবণতা দীর্ঘস্থায়ী হয়নি। মুসলমানদের শিক্ষিত ও সচেতন অংশ বঙ্গবিভাগের প্রকৃত কর্মপরিকল্পনার সঙ্গে সঙ্গে বঙ্গকে স্বাগত জানায়। ২৮ মুসলমান নেতৃবৃন্দ নতুন লে. গভর্নর ব্যামফিল্ড ফুলারকে উষ্ণ সংবর্ধনা জ্ঞাপন করে। 'মোসলেম ক্রনিকল’ পত্রিকা এক বৎসর বঙ্গবিভাগের বিরোধীতা করলেও পরে বঙ্গভঙ্গের সিদ্ধান্তকে সমর্থন করে।

নোহানেডান লিটারেরি সোসাইটির সাতজন বিশিষ্ট মুসলমান ব্যক্তির স্বাক্ষরিত একটি লিখিত ঘোষণা পূর্ব ও পশ্চিম বাংলার বিভিন্ন মুসলমান সোসাইটির নিকট বিলি এবং বঙ্গভঙ্গের সিদ্ধান্তকে শর্তহীন সমর্থন প্রদানের জন্য অনুরোধ জানায়।২৯ নতুন প্রদেশের মুসলমানদের সামাজিক, রাজনৈতিক ও অর্থনৈতিক আশা-আকঙ্খা বাস্তবায়নের উদ্দেশ্যে ১৯০৫ সালের ১৬ অক্টোবর ‘মোহামেডান 
প্রভিন্সিয়াল ইউনিয়ন' নানে একটি সংগঠন প্রতিষ্ঠা করা হয়। খাজা সলিমুল্লাহঃ০ সর্বসম্মতিক্রনে এর পৃষ্ঠপোষক মনোনীত হন। ১৯০৬ সালে প্রতিষ্ঠিত নিখিল ভারত মুসলিমস লীগ বঙ্গভঙ্গের প্রতি সমর্থন জ্ঞাপন করে। বাঙ্গালি মুসলমান পত্র-পত্রিকাগুলো বঙ্গভঙ্গের প্রতি সমর্থন জানায়। ‘ইসলাম প্রচারক’ পত্রিকা স্বদেশী আন্দোলনকে হিন্দুদের স্বার্থে আন্দোলন হিসেবে অভিহিত করেন। ‘আঞ্জুমানে মফিদুল ইসলাম’ এর পৃষ্ঠপোষকতায় একটি লাল পুস্তিকা প্রকাশ করে হিন্দুদের সাথে সব ধরনের সংযোগ ছিন্ন করার জন্য বাংলা ও আসানের মুসলমানদের প্রতি আহ্বান জানানো হয়। এসব প্রচারণার ফলে অবস্থা এমন পর্যায়ে পৌঁছে যে ১৯০৭ সালের মার্চ মালে কুমিল্লায় ও এপ্রিল মালে জামালপুরে হিন্দু-মুসলমানদের মধ্যে সাম্প্রদায়িক দাঙ্গা সংঘটিত হয়।

\section{বঙ্গভঙ্গের ফলাফল}

বাংলার ইতিহালে ১৯০৫ সালের বঙ্গভঙ্গ একটি গুরুত্বপূর্ন ঘটনা। তবে এটা স্বীকার্য যে, ১৯০৫ পর্যন্ত বিভিন্ন আন্দোলনগুলির মধ্যে বঙ্গভঈ একমাত্র আন্দোলন যার ব্যাপকতা ছিল, আন্দোলনের পক্ষে বিপক্ষে অংশগ্রহণকারী মানুযের সংখ্যা ও ছিল বেশি। পরবর্তীতেকালে আরও ব্যাপক ভিত্তিতে গড়ে ওয় স্বদেশী বা স্বরাজ আন্দোলনের ভিত্তি ছিল বঙ্গভঙ্গের আবেগজাত উপাদানাবলী যা ঔপনিবেশিক সরকার বিরোধী আন্দোলনে পরিণত হয়েছিলো ।১s

বঙ্গভঙ্গ ঘটনাই বাংলার হিন্দু ও মুসলমান জনগোষ্ঠীকে আলোড়িত করেছিল এবং বাংলা তথা ভারতের পরবর্তী রাজনৈতিক ঘটনাবলীকে প্রভাবান্বিত করেছিল। বঙ্গঞ্দের উদ্যোগ প্রশাসনিক কারণে নেয়া হনেও পরবর্তীকালে রাজনৈতিক উল্দেশ্য- অর্থাৎ হিন্দু ও মুসলমান সম্প্রদায় দুটিকে বিভক্ত করে। শাসন করার নীতি (Divide and Rule) যুক্ত হয়। বঙ্গভঙ্গের প্রস্তাব উত্থাপিত হওয়ার সময় থেকে বঙ্গভঙ্গ বিরোধী যে আন্দোলনের সূত্রপাত হয় তা বঙ্গভঙ্গের রদ না হওয়া পর্যন্ত চলে। আন্দোলনের কেন্দ্র ছিল কলকাতা এবং আন্দোলনের ঢেউ সারা বাংলায় ছড়িয়ে পড়েছিল। আন্দোলনটি মূলতঃ ছিল শহুরে এবং তার নেতৃত্ব ছিল হিন্দু ভদ্রলোক শ্রেণীর হাত। নিজ নিজ স্বার্থ চিন্তা থেকে বিভিন্ন পেশার হিন্দু নেতৃবৃন্দ আন্দোলনে অংশগ্রহণ করনে ও তাঁরা আন্দোলনের কারণ হিসেবে বাংলা ভাষাভাষিদেরকে বিভক্ত করার চক্রান্তের বিরুদ্ধে প্রতিবাদ হিসেবে প্রচার করেছেন। তবে বঙ্গভঙ বিরোধী আন্দোলন বাংলার সাম্প্রদায়িকতার বীজ বপন করে। বঙ্গভঙকে অধিকাংশ মুসলমান স্বাগত জানালেও অধিকাংশ হিন্দু তার বিরোধিতা করে। ফনে হিন্দু-মুসলমানের সম্পর্কে ফাটল ধরে। জনুালাভ করে মুসলমানদের আলাদা রাজনৈতিক সংগঠন হিসেবে মুসলিম লীগ। বঙ্গভঙ্গ বিরোধী আন্দোলন থেকে স্বরাজ প্রতিষ্ঠার দাবী সুচিত হয়। ছাত্ররা রাজনীতিতে জড়িয়ে পড়ে। শ্রমিক শক্তির উত্থান ঘটে। আবার বঙ্গঞ্গের ঘটনা মুসলমানদেরকে আত্নসচেতন করে, উন্মোষ ঘটে মুসলিম জাতীয়তাবাদের হিন্দু ও মুসলমান সম্প্রদায়ের সম্পর্কের মধ্যে সেই যে ফাটল ধরে, তা আর কখনই জোড়া লাগেনি। বঙ্গ বিভাগ যদি না হতো তাহলে বঙ্গালি জাতি দক্ষিণ এশিয়া তথা সমগ্র পৃথিবীতে পরাক্রমশীল জাতি হিসেবে নিজেদের অবস্থান ধরে রাখত।

\section{উপসংহার}

ব্রিটিশ বণিকেরা বাণিজ্যের মাধ্যমে ভারত উপমহাদেশে প্রবেশের মধ্য দিয়ে নিজেদের প্রাধান্য বিস্তার করে। পরবর্তীকালে ১৭৫৭ সালে বাংলার শেষ স্বাধীন নবাব সিরাজ-উদ-দৌল্লাকে পরাজিত করে, বাংলার দেওয়ানি রাজস্ব গ্রহণ করে। বাংলার দেওয়ানি রাজস্ব গ্রহণ করে প্রশাসনিক প্রভাব বিস্তার করে, তৎকালীন ব্রিটিশ রানী এলিজাবেথ এই ব্রিটিশ বাণিজ্য গোষ্ঠীকে নিয়ন্ত্রণের জন্য ব্রিটিশ সিভিলিয়ান তথা তৎকালীণ আমলাদের পাঠায়। তারা এই প্রশাসনিক ক্ষমতা গ্রহণের মাধ্যনে বাংলাকে ১৯০৫ সালে বাংলাকে বিভক্ত করার নীতি গ্রহণ করে শাসন ক্ষমতার বৃদ্ধির প্রয়াস চালায়। এই ফলশ্রততিতে বাংলায় দুই প্রধান গোষ্ঠী হিন্দুমুসলিম বিভিন্ন আন্দোলনে জড়িয়ে পড়ে। যদিও বা বঙ্গো প্রদেশ একসময় ভাষার ভিত্তিতে এক ছিল। কিন্তু ১৯০৫ সালে বঙ্গভঙ্গ এই বৃহৎ প্রদেশকে দ্বিখন্ডিত করে নানা সমস্যার সৃষ্টি করে। একদিকে উচ্চ বিত্ত হিন্দু শ্রেণী নিজেদের স্বার্থ রক্ষার্থে বঙ্গ বিভাগের বিরোধীতা করে অন্যদিকে তৎকালীন অধিকার বঞ্চিত মধ্যবিত্ত মুসলিম শ্রেণী নতুন ও স্বতন্ত্র রাজনীতি করার আসায় এই বঙ্গভঙ্গকে স্বাগত জানায়। তবে বঙ্গঙ্গের উদ্য্যাগটি প্রথনে ছিল প্রশাসনিক কিন্তু এর সঙ্গে পরে যুক্ত হয় রাজনৈতিক সুবিধা লাভের চিন্তা। বঙ্গভঙ্গ প্রস্তাব দেয়া থেকে বঙ্গ কার্যকর হওয়া পর্যন্ত সারা বাংলায় বঙ্গঙ্গবিরোধী আন্দোলন গড়ে ওঢে। আন্দোলনের কেন্দ্র ছিল কলকাতা। আন্দোলনকারীদের কৃতিত্ব এই যে, প্রথমবারের মতো সারা বাংলায় তারা সমভাবে একটি আন্দোলন গড়ে তোলায় পদ্ধতি টৈরি করেছিলেন যা ছিল শৃজ্খলাপূর্ণ। বঙ্গভঙ্গ আন্দোলন জনমত সংগঠনের একটি নেটওয়ার্ক তৈরির পদ্ধতিতে অভিজ্ঞ করে তুলেছিলো রাজনীতিবিদদের যা তারা পরে ব্যবহার করতে পেরেছিলেন বাংলার বিভিন্ন আন্দোলনে।

\section{গ्रন্থ পুঞ্টি}

১। বঙ্গভঙ্গ সম্পর্কে বিস্তারিত দ্রষ্টব্যঃ

Sufia Ahmed, Muslim community in bengal 1884-1912, Dacca: oxford university press, 1974; Ajit k. Neogy, Partitiors of Bengal, Calcutta: A Mukherjee \& Co. Pvt. Ltd. 1987; MKU Molla, The New Provinces of Eastern Bengal and Assam, অর্জুন গোস্বামী (সম্পাদিত), বঙ্গ ১৯০৫, কলকাতা: চয়নিকা, ২০০৫; (ড.) কে.এম. মোহসীন ও অন্যান্য (সম্পাদিত) বঙভঙ্গের শতবর্ষ, ১৯০৫-২০০৫ ঢাকা: প্রেস উইং, বঙভবন ২০০৬; মনতাসীর মামুন, ১৯০৫ সালের বঙ্গ পূর্ববল্েে প্রতিক্রিয়া, ঢাকা: মাওলা ব্রাদার্স, ১৯৯৯; মুনতাসীর মামুন, বঙ্গ, ঢাকা: সমাজ নিরীক্ষণ কেন্দ্র, ১৯৮-১। 
२। S. Razi Wasti, 'partition of Bangal and its immediate Effects; Journal of the Asiatic Society of Pakistan, Vol. XI, No. 1, April 1966, PP. 63-72

৩। John R. Mclane, 'The decision to partition in 1905; Economic and social History Review, Vol. 11, No. 3, July 1965.

৪। অমলেশ ত্রিপাঠি, ভারতের মুক্তিসংগ্রামের চরমপন্থী পর্ব, (অনুবাদ: নির্মল দত্ত), কলকাতা: ১৯৮৭, তৃতীয় অধ্যায়।

৫। দিলীপ কুমার সাহা, দক্ষিণ এশিয়ার ইতিহাস (১৫২৬ খ্রীঃ হতে ১৮৫৭ খ্রীঃ পর্যন্ত) পৃ: ২৭৭

৬। বঙ্গভঙ্গের প্রশাসনিক কারণের পক্ষে লিখেছেন কে.এম. মোহসীন, দেখুন তার প্রবন্ধ, কে.এম. মোহসীন, ‘বঙ্গভঙ, বঙ্গভঙ্গ।

१। Sir Andrew H.L. Fraser, Among Indian Rajhas and Ryotes, allahabad, 1975 PP. 323-324।

৮। ।দদৃৃত: এমাজউদ্দীন আহমদ, ১৯০৫ সালের বঙ্গবিভাগ: একটি রাজটৈতিক পদক্ষেপ।

৯। উদৃত: এমাজউদ্দিন আহমদ, পূর্ব্বাক্ত প্রবন্ধ বঙ্গভঙ্গ।

১০।

১১। দেখুন, Lovat Fraser, India Under carzon and After, London, 1911

১২। দেখুন, Lovat Fraser, India Under carzon and After, London, 1911, PP.323

১৩। এমাজউদ্দীন আহমদ, প্রাগুক্ত থ্রবন্ধ, পৃ: ১৯

১8।

১৫। ঐ, প: ১৩

১৬। ঐ, পৃ: ২১

১৭। ড. মোঃ মাহবুবর রহমান, বাংলাদেশের ইতিহাস; ১৯০৫-৪৭, পৃ: ২৬

১৮-। উদ্থত, সুমিত কুমারের প্রাগুক্ত প্রবন্ধ, পৃঃ ১৭-১৮-

১৯। The Bengale, 13.12.1903; সুরেন্দ্র নাথ ব্যানার্জি লেখেন, 'We object to the Proposed dismemberment of Bengal and we are sure the whole country will rise as one man to protest againt it;

২०।

২১। সে সময় অধিকাংশ পত্র পত্রিকার মালিক ছিলেন হিন্দু জমিদার বা মধ্য বিত্ত শ্রেণীর। স্বাভাবিকভাবেই তাদের বঙ্গভঙ্গ বিরোধী মনোভাবই প্রতিফলিত হয়েছে ঐসব পত্র-পত্রিকায়।

২২। মুনতাসীর মামুন, ১৯০৫ সালের বঙ্গঙ, প্রাগুক্ত, পৃ. ১৭।

২৩। ঐ, পৃ. ১৯।

২8। ঐ, পৃ. ১৮-।

২৫। Sufia Ahmed, Op. cit 255-256 এ.টি.এম. আতিকুর রহমান ‘বঙ্গঙ্গ বিরোধী আন্দোলনে মুসলমানের একটি পর্যালোচনার অর্জুন গোস্বামী (সম্পা), বঙ্গভঙ্গ ১৯০৫, কলকাতাः চয়নিকা, ২০০৫।

২৬। Sufia Ahmed, Op.cit; PP. 246 মুনতাসীর মামুন, প্রাগুক্ত, পৃ. ১৯।

২৭। মুনতাসীর মামুন, ১৯০৫ সালের বঙ্গঙ, শ্রাগুক্ত, পৃ. ৩০।

২৮-। Sufia Ahmed, Op.cit; PP. 252;

২৯। Mku Molla, 'Keir Hardic and the first partition of Bengal' 'Rajshahi University Studies, Vol. III, January 1970, appendix B, P. 108; মুনতাসীর মামুন, ১৯০৫ সালের বঙভঙ প্রাগুক্ত পাদটিকা নং ৬৫. পৃ. ৩৬।

৩০। Sufia Ahmed, Op. cit; PP. 246।

৩১। মুনতাসীর মামুন, ১৯০৫ সালের বঙভঙ, প্রাগুক্ত, পৃ. ৩০। 


\section{Publish Online and Print Version Both}

Online ISSN: 2312-2021

Google Scholar: https://goo.gl/eozEWi 